\title{
Minimally invasive endoscopic treatment of necrotizing pancreatitis: A case report with images and review of the literature
}

\author{
Cassia lemos Moura ${ }^{1}$, Priscila Pulta Azevedo Barros ${ }^{1}$, Cristiane Mota Oliveira ${ }^{1 *}$, Rogerio Colaiácovo², Julana Marques \\ Drigo ${ }^{2}$, Augusto Pincke Cruz Carbonarl ${ }^{2}$, Tercio de Campos $^{3}$, Lucio Giovani Batista Rossini ${ }^{4}$ \\ ${ }^{1}$ Endoscopy Fellow, Endoscopy Department, Santa Casa de São Paulo, São Paulo, SP, Brazil \\ ${ }^{2}$ Assistant Physician, Centro Franco-Brasileiro de Ecoendoscopia (CFBEUS), Santa Casa de São Paulo, São Paulo, SP, Brazil \\ ${ }^{3}$ Assistant Physician, Surgery Department, Santa Casa de São Paulo, São Paulo, SP, Brazil \\ 'Head Physician, CFBEUS, Santa Casa de São Paulo, São Paulo, SP, Brazl
}

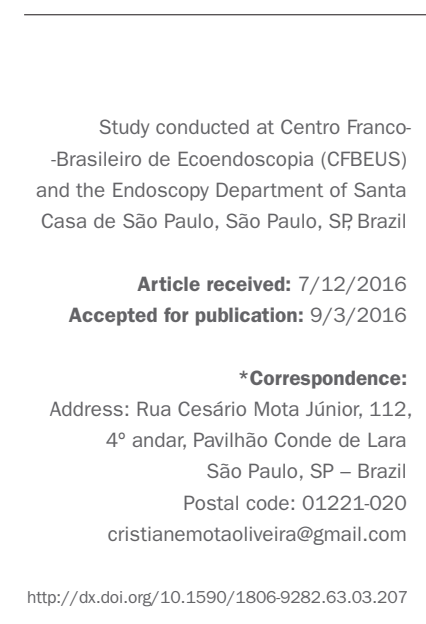

\section{SUMmARY}

Necrotizing pancreatitis with fluid collections can occur as a complication of acute pancreatitis. The management of these patients depends on the severity and involves multiple medical treatment modalities, as clinical intensive care and surgical intervention. In this article, we show a severe case of walled-off pancreatic necrosis that was conducted by endoscopic drainage with great clinical outcome.

Keywords: acute pancreatitis, necrotizing pancreatitis, endoscopic ultrasound.

\section{INTRODUCTION}

Acute pancreatitis is a common and potentially lethal disease. Approximately $20 \%$ of these patients develop necrotizing pancreatitis, which is associated with a $15 \%$ mortality rate. ${ }^{4}$ In 2012, the term "walled-off necrosis" (WON) was proposed, which is based on the appearance of pancreatic or peripancreatic necrosis, typically arising four weeks after the initial symptoms. This necrosis is heterogeneous, contains solid material with varying amounts of fluid and has an encapsulated wall. ${ }^{2}$ Typically, the progression of the disease is accompanied by clinical and laboratory worsening. ${ }^{1}$

Recognizing these patient's severity, less invasive treatment strategies have been advocated, such as percutaneous drainage, endoscopic treatment and, if necessary, minimally invasive surgical necrosectomy.

On the other hand, currently, it has been applied less invasive treatment strategies, as percutaneous drainage, minimally invasive surgery and endoscopic treatment.

\section{Case}

A female patient, 36 years old, admitted to our hospital presenting epigastric pain irradiating to the back, which started a day before. Laboratory studies have shown leukocytosis with a left shift, serum amylase of 2,086, and increased GGT (GGT: 589 AF: 204). Abdomen computed tomography $(\mathrm{CT})$ scan performed 72 hours after admission has identified extensive pancreatic necrosis with fluid accumulation (Balthazar 9) (Figure 1). The patient was initially treated with broad spectrum antibiotics; however, the disease progressed with clinical and laboratorial worsening, demonstrated by leukocytosis and C-reactive protein (CRP) increases, hemoglobin drop, acute renal failure and need of intensive care unit, where the patient remained under vasopressor and ventilator support. A control CT scan held after 10 days identified necrotic collection occupying more than $50 \%$ of the pancreatic parenchyma and an increase of the retroperitoneal free liquid (Figure 2).

Given the severity of the case, the medical team decided to perform a peripancreatic collection endoscopic drainage four weeks after admission. First, the purulent cavity was delimited by endoscopic ultrasound (EUS). Then, ultrasound-guided transmural drainage was conducted using two $7 \mathrm{Fr}$ plastic prosthesis in the gastric body (Figure 3).

The patient progressed with significant improvement and was discharged 30 days after the procedure. After 90 days, she returned to the hospital in order to remove the plastic prosthesis endoscopically. The patient is currently under clinical, radiological and laboratorial monitoring, which proved the effectiveness of the treatment. 


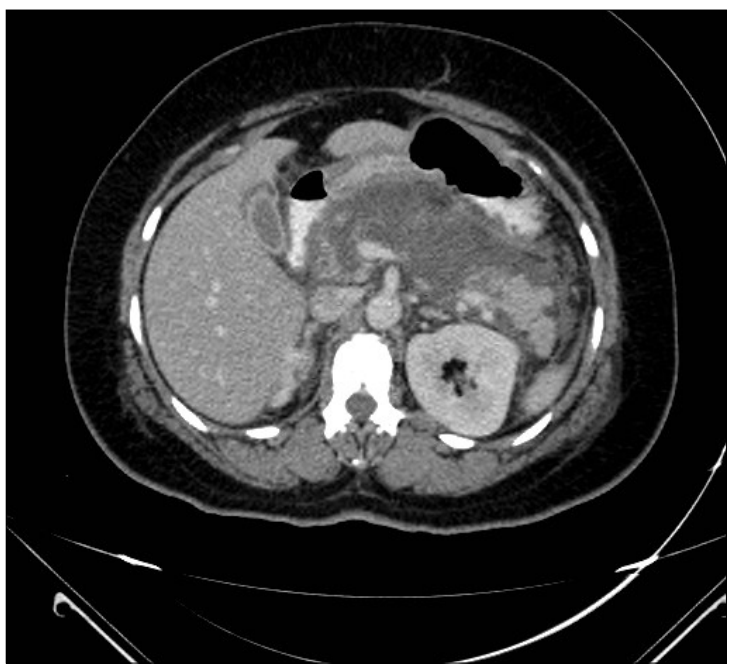

FIGURE 1 CT scan 48 hours after admission, showing edematous acute pancreatitis.

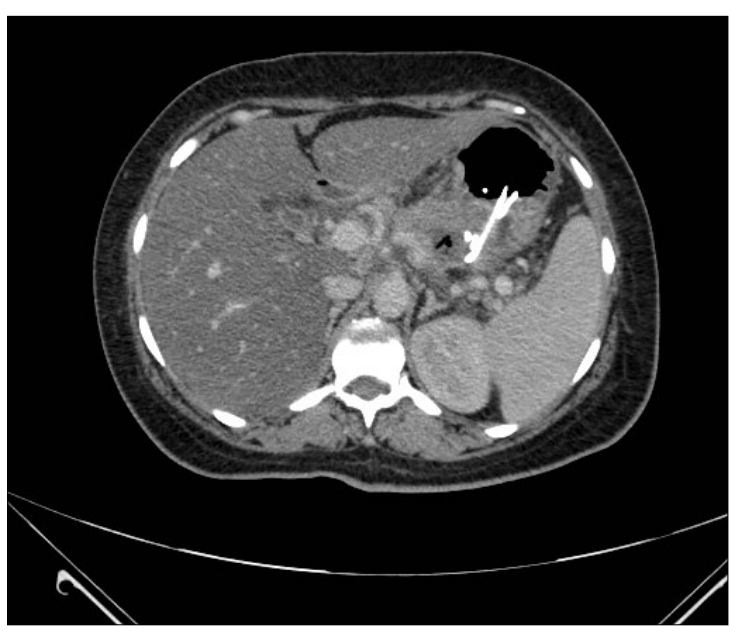

FIGURE 3 TC scan with plastic prosthesis positioned at the posterior wall of the stomach draining the necrotic cavity (WON).

\section{Discussion}

The so-called Atlanta classification was proposed in 1992 in order to standardize the severity of acute pancreatitis and its complications. ${ }^{1-3}$ In 2012, it was revised, and pancreatic inflammatory collections were reclassified. Acute fluid collections include those which are not completely encapsulated and which appear within four weeks from the initial presentation.

These collections can be intra or extra-hepatic and may contain solid material with varying amounts of liquid. Chronic collections are those occurring after four weeks from the initial presentation and include pancreatic pseudocysts and WON. Pseudocysts usually develop adjacent to the pancreas, and they are homogeneous and filled with fluid.

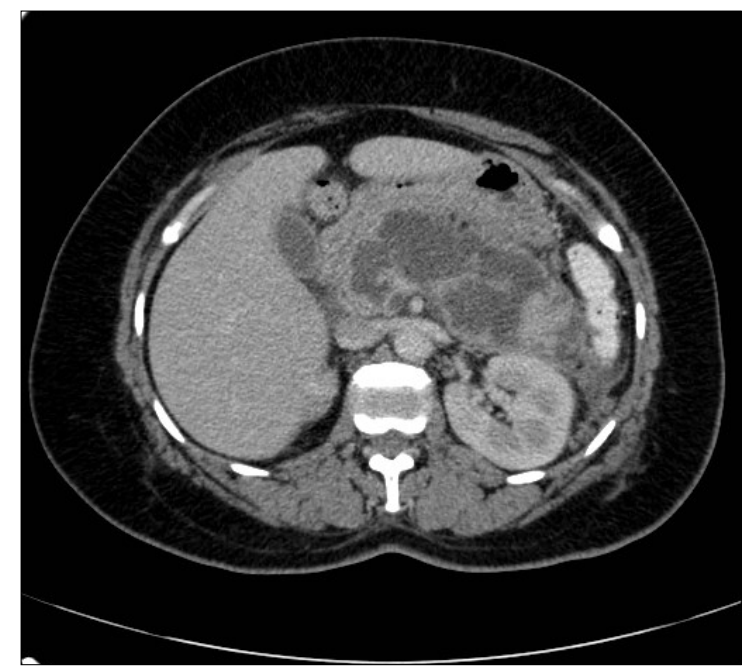

FIGURE 2 CT scan performed 10 days after admission, showing fluid collection and necrosis (WON).

Walled-off necrosis, in turn, occurs in about $1-9 \%$ of all cases of severe acute pancreatitis, late in the disease course (after four weeks) and consists in a circumscribed area containing thick liquid and pancreatic necrotic debris, which replaces part of the pancreatic parenchyma $a^{2,3}$ and originates from the necrotic area. These radiological findings can be noticed on CT scan, and therefore are best characterized by magnetic resonance imaging (MRI). These findings are typically accompanied by clinical and laboratory worsening. ${ }^{1}$

Endoscopic ultrasound has emerged as a minimally invasive procedure, with low risk of complications, allowing the complete drainage of pancreatic or peripancreatic collections. The procedure is performed under real time imaging with the use of Doppler if necessary to prevent iatrogenic complication. Drainage is done by carrying out a fistula between the gastrointestinal tract (stomach and duodenum) and the retroperitoneal cavity, followed by the placement of plastic stents. ${ }^{4}$

Endoscopic treatment can be performed through the opening of a gastrointestinal tract fistula (stomach and duodenum) with the retroperitoneal cavity containing liquid, followed by placement of a prosthesis in order to drain the contents. ${ }^{4}$ The ideal puncture place can be predetermined with the aid of endoscopic ultrasound, especially in collections in the tail, and in patients with varicose veins. The most appropriate puncture sites are in walls not ticker than $10 \mathrm{~mm}$. If the EUS is not used, the reference point will be the external compression of the gastric or duodenal wall, shown endoscopically. The integrity of the pancreatic duct can be assessed by CT, MRI, EUS or endoscopic retrograde cholangiopancreatography (ERCP). ${ }^{2}$ 
In patients undergoing EUS, transmural puncture is directly guided, with the aid of color Doppler in order to avoid wall vascular injuries that may occur while puncturing. Cavity content aspiration and/or contrast injection demonstration confirms fluoroscopic guiding. ${ }^{2}$

Drainage is traditionally performed with a plastic prosthesis, given that one or two prostheses of varying sizes can be placed (7-10F). These stents with small diameters make proper drainage difficult. In cases of WON, early stent obstruction, infection and multiple endoscopic therapies might be necessary. ${ }^{5}$

Recently, a fully covered-self expanding metal stent with double anchoring has been developed. This type of stent has shown to be more effective and safe to perform endoscopic transmural drainage. In cases of WON, stent dilation performed using a balloon allows the achievement of larger diameters (10 or $15 \mathrm{~mm}$ ), leading to a more effective cavity drainage and easier access for possible futures endoscopic necrosectomies. ${ }^{5}$

Recent studies have claimed that endoscopic necrosectomy has reduced pro-inflammatory post-procedural response, which can be measured by serum interleukin- 6 increases. Therefore, the number of intra-abdominal bleedings, enterocutaneous and pancreatic fistula, multiorgan system failure and death has decreased. ${ }^{2}$ Furthermore, endoscopic treatment avoids laparotomy or lobotomy and their complications. ${ }^{2}$

According to recent studies, the most common complication of endoscopic drainage is hemorrhage, reported in about $9 \%$ of the cases. However, this complication has been successfully treated by endoscopic management with epinephrine injection and hemostatic clips. ${ }^{2}$ Other less common complications are pancreatic fistula, ${ }^{4}$ prosthesis migration/ occlusion $^{1}$ and pneumoperitoneum/perforation, which have been conducted conservatively. However, all related complications were conducted conservatively. A single case of embolism has been reported. ${ }^{2}$ Other studies have reported complications such as infection (particularly in undrained necrosis), pancreatitis, stent migration or occlusion, pancreatic duct injuries and complications from sedation. ${ }^{4}$

Although endoscopic treatment is considered safe for necrotizing pancreatitis, some questions are yet to be answered. ${ }^{4}$ To date, there is still no consensus on what kind of necrosis should be treated this way. In addition, time of intervention, indication and treatment steps have not been well-established. ${ }^{2}$ Therefore, therapy can start either with endoscopy or drainage. If this first intervention does not succeed or is not sufficient, the procedure can be repeated or performed with an alternative adjunct technique. ${ }^{2}$
In conclusion, we described a relevant case of severe acute pancreatitis progressing with WON that was successfully treated with a single procedure of transmural endoscopic drainage. Some important issues should be noticed, such as the clinical severity and the decrease in inflammatory response achieved with exclusive endoscopic drainage.

In the reported case, $7 \mathrm{Fr}$ plastic prosthesis was the only one available at our institution at that moment. Despite the lack of a self expanding metal stent with double anchoring, the drainage performed has shown to be satisfactory.

Treatment must be performed by experienced professionals and the availability of resources and maintenance of organ function must be considered. Until now, there is no gold standard therapy and the best treatment must involve a multimodal adaptive perspective. ${ }^{2}$

Currently, endoscopic therapy has been shown to be effective. However, further prospective and randomized studies allowing comparison of endoscopic therapy and percutaneous drainage, laparoscopy and open surgery are needed. ${ }^{2}$

\section{Resumo}

Tratamento endoscópico minimamente invasivo: relato de caso e revisão da literatura

Pancreatite necrosante com coleções pode ocorrer como complicação da pancreatite aguda. O manejo desses pacientes depende da gravidade e envolve múltiplas modalidades médicas de tratamento, como terapia clínica intensiva e intervenção cirúrgica. Neste artigo, mostramos um caso grave de necrose pancreática com ótima resolução clínica após drenagem endoscópica.

Palavras-chave: pancreatite aguda, pancreatite necrotizante, ecoendoscopia.

\section{References}

1. ASGE Standards of Practice Committee, Muthusamy VR, Chandrasekhara $\mathrm{V}$, Acosta RD, Bruining DH, Chathadi KV, et al. The role of endoscopy in the diagnosis and treatment of inflammatory pancreatic fluid collections. Gastrointest Endosc. 2016; 83(3):481-8.

2. Gardner TB. Endoscopic management of necrotizing pancreatitis. Gastrointest Endosc 2012; 76(6):1214-23.

3. Voermans RP, Fockens P. Endoscopic transluminal drainage of walled-off necrosis: does size matter? Gastrointest Endosc. 2016; 83(4):708-10.

4. van Brunschot S, Fockens P, Bakker OJ, Besselink MG, Voermans RP, Poley JW, et al. Endoscopic transluminal necrosectomy in necrotizing pancreatitis: a systematic review. Surg Endosc. 2014; 28(5):1425-38.

5. Siddiqui AA, Adler DG, Nieto J, Shah J, Binmoeller KF, Kane S, et al. EUS guided drainage of peripancreatic fluid collections and necrosis by using a novel lumen-apposing stent: a large retrospective, multicenter U.S. experience. Gastrointest Endosc. 2016; 83(4):699-707. 